\title{
Critical Behavior of Low Dimensional Magnetic Systems
}

\author{
Aycan Özkan and Bülent Kutlu* \\ Gazi Üniversitesi, Fen Fakültesi, Fizik Bölümü, \\ 06500 Teknikokullar, Ankara, Turkey. \\ e-mail: aycan@gazi.edu.tr \\ *e-mail: bkutlu@gazi.edu.tr
}

November 6, 2018

\begin{abstract}
In this study, critical behavior of low dimensional magnetic systems as cyano-bridged $\mathrm{Tb}$ (III)-Cr(III) bimetallic assembly was investigated with the mixed spin 3- spin 3/2 Ising model. The mixed spin Ising model is simulated with Cellular Automaton cooling and heating algorithms on one-dimensional lattices in periodic boundary conditions. The Ising model Hamiltonian includes only antiferromagnetic nearest-neighbor interaction $(J>0)$. The mixed spin system behaves like the isolated one-dimensional chain for zero magnetic field $\left(h=\frac{H}{J}=0\right)$. In the presence of the magnetic field, the magnetization is calculated using zero-field cooling $(Z F C)$ and field cooling $(F C)$ processes. The one-dimensional Ising model results are compatible with the cyano-bridged $\mathrm{Tb}(\mathrm{III})-\mathrm{Cr}(\mathrm{III})$ bimetallic quasi-one dimensional assembly $\left(\left(\left[\mathrm{Tb}\left(\mathrm{H}_{2} \mathrm{O}\right)_{2}(\mathrm{DMF})_{4}\left\{\mathrm{Cr}(\mathrm{CN})_{6}\right\}\right] \cdot \mathrm{H}_{2} \mathrm{O}(\mathrm{DMF}=\right.\right.$ dimethylformamide)) results.

Key words: Hysteresis, long-range order, Ising model, cellular automaton.

PACS Numbers: 05.20.-y, 75.10.Hk, 05.10.-a, 75.60.-d.
\end{abstract}

\section{Introduction}

Low dimensional magnetism has been a subject of studies for many years. In the last decades, new materials have been synthesized to obtain high temperature magnetism. One of these materials are cyano-bridged $4 f-3 d$ assemblies. $f$-block lanthanide ions having large anisotropic magnetic moments yield hard magnets and long-range magnetic order in solids [4, 9-11]. Some of cyanobridged $4 f-3 d$ assemblies also exhibit field-induced magnetic relaxation [10], cooling-rate dependent magnetism [12], photo-induced magnetization [13], and humidity response [14] . Interactions between the ions/molecules determine the 
electronic and magnetic properties as well as dimensionality of the assembly. Guo et al. synthesized cyano-bridged $\mathrm{Tb}$ (III) - Cr (III) bimetallic assembly $\left(\left[\mathrm{Tb}\left(\mathrm{H}_{2} \mathrm{O}\right)_{2}(\mathrm{DMF})_{4}\left\{\mathrm{Cr}(\mathrm{CN})_{6}\right\}\right] \cdot \mathrm{H}_{2} \mathrm{O}(\mathrm{DMF}=\right.$ dimethylformamide $\left.)\right)$ [4]. They introduced that antiferromagnetic interaction between $\mathrm{Tb}$ (III) and $\mathrm{Cr}$ (III) ions represented by $S=3$ and $\sigma=3 / 2$, respectively, leads to ferrimagnetic structure in the quasi-one dimensional zig-zag chain. A transition to 3D longerange magnetic order from the ferrimagnetic $\mathrm{Tb}$ (III) - Cr (III) chains occurs at $T_{C}=5 K$ with the weak interchain interactions. Therefore, they draw attention to the requirement of the further experimental and theoretical studies to illuminate the magnetic interaction mechanism.

The aim of this study was to detect the interaction mechanism of the onedimensional spin 3 - spin $3 / 2$ chain. For this purpose, the one-dimensional spin 3 - spin 3/2 Ising model in its simplest form is simulated using Cellular Automaton (CA) and the results are compared with the experimental results to clear up the magnetic interaction mechanism. The one-dimensional Ising model was first introduced by Ernst Ising in 1925. The model established by Ising as a chain of spins, each spin interacts only with its nearest-neighbors, and an external field. At non-zero temperature, the model does not have any phase transition. Correlation lenght becomes infinite at $H=T=0$, which is the critical point of the model [1]. However, magnetic order can emerge with broken one-dimensionality due to orbital degeneracy or quasi-one dimensional geometry $[2-8]$.

The mixed spin Ising model is a simple model to study ferrimagnetism. Therefore, a variety of spin mixtures, such as spin $1-$ spin $1 / 2$ [15-22], spin 1 - spin $3 / 2[23-25]$, spin 1 - spin $5 / 2$ [26], spin 2 - spin $1 / 2$ [15], spin 2 spin $3 / 2[27,28]$, spin 2 - spin $5 / 2$ [29 - 34], spin $1 / 2$ - spin $3 / 2$ [15,21,35], spin $1 / 2$ - spin $5 / 2$ [15], spin $3 / 2$ - spin $5 / 2$ [17], and spin 3 - spin $3 / 2$ [36] have been studied frequently by simulation and numerical methods. Creutz Cellular Automaton (CCA) algorithm and its improved versions are efficient to study the critical behaviors of the Ising model $[36-40]$. The CCA algorithm was first introduced by Creutz [41]. It is a microcanonical algorithm interpolating between the conventional Monte Carlo and the molecular dynamics techniques.

In this study, magnetization $(M)$, susceptibility $(\chi)$, internal energy $(U)$, and specific heat $(C / k)$ are calculated on one-dimensional chain of linear dimension $L=100,500,1000,5000,10000,50000$, and 100000 with periodic boundary conditions. First, $1 D$ behavior and the long-range order $(L R O)$ of the mixed spin system have been investigated with temperature variation of the thermodynamic quantities in zero external field $\left(h=\frac{H}{J}=0\right)$ and external field ( $\left.h=\frac{H}{J} \neq 0\right)$ using the Cellular Automaton cooling algorithm. At the same time, the thermodynamic quantities are calculated via field cooling $(F C)$ and zero field cooling $(Z F C)$ processes for $0 \leq h \leq 3.4$. For mixed spin systems, hysteresis curves are obtained at several temperature values. The outline of this paper is as follows: In Section 2, the model and the formalism are given. In Section 3, the results and the discussions are presented. A conclusion is given in Section 4. 


\section{Model}

The mixed-spin Ising model hamiltonian is given by

$$
H_{I}=J \sum_{<i j>} S_{i} \sigma_{j}-H \sum_{i}\left(S_{i}+\sigma_{i}\right)
$$

where $S_{i}=0, \pm 1, \pm 2$ and \pm 3 and $\sigma_{j}= \pm 1 / 2, \pm 3 / 2 .<i j>$ denotes the summation over all nearest -neighbour spin pairs in a one-dimensional lattice. $J$ is the bilinear interaction $(J>0)$ between $S$ and $\sigma . H$ is the external field. The lattice is established from the two interpenetrating linear chains named as sublattice $A$ and sublattice $B . S$ and $\sigma$ spins are located in sublattice $A$ and sublattice $B$, respectively (Fig. 1). Three variables are associated with each site of the lattice. The values of these variables are determined in each site from its value and those of its nearest- neighbors at the previous time step. The updating rule, which defines a Cellular Automaton, is as follows: Of the three variables on each site, the first one is the Ising spin, $A_{i}$ or $B_{j}$. Its values may be $A_{i}=0,1,2,3,4,5$, and 6 for $S$ and $B_{j}=0,1,2$, and 3 for $\sigma . S$ and $\sigma$ can be defined as $S_{i}=\left(A_{i}-3\right)$ and $\sigma_{j}=\left(2 B_{j}-3\right) / 2$ using the Ising spin variables in Eq. (1). The second variable corresponds the momentum variable which is conjugate to the spin (the demon). The kinetic energy associated with the demon, $H_{K}$, is an integer and it is equal to the change in the Ising spin energy $\left(-d H_{I}\right)$ for any spin flip.

$$
d H_{I}=H_{I}^{t}-H_{I}^{t+1}
$$

Kinetic energy values lie in the interval $(0, m)$ where $m$ takes a different value for each $h=\frac{H}{J}$. For example, the greatest value of the $d H_{I}$ equals -24 for $J=1$ and $H=1$. In those terms, $m$ equals 48 .

The total energy $(T E)$ which is conserved is given in the following form:

$$
T E=H_{I}+H_{K}
$$

The third variable provides a checkerboard row style updating and so it allows the simulation of the Ising model on a cellular automaton. The black sites of the checkerboard are updated and then their color is changed into white; white sites are changed into black without being updated. The updating rules for the spin and the momentum variables are as follows: For a site to be updated its spin is changed to one of the other $6(3)$ states with $1 / 6(1 / 3)$ probability for $S(\sigma)$ and the change in the Ising spin energy $d H_{I}$ is calculated. If this energy change is transferable to or from the momentum variable associated with this site, such that the total energy $T E$ is conserved, then this change is done and the momentum is appropriately changed. Otherwise, the spin and the momentum are not changed. For example, $d H_{I}$ equals 24 in the case of $\sigma_{i}^{t}=-\frac{3}{2}, S_{i}^{t}=-3$, and $\sigma_{i+1}^{t}=-\frac{3}{2} . S_{i}^{t+1}$ can take one of the $S_{i}=3,2,1,0,-1,-2$, and -3 values at $t+1$ time step If the $S_{i}^{t+1}$ takes the value of 3 , the 24 unit energy is transferred to the system as the kinetic energy. 
The system temperature for a given total energy is obtained from the average value of the kinetic energy, which is given by

$$
\langle E\rangle=\frac{\sum_{n=0}^{m} n e^{-n J / k T}}{\sum_{n=0}^{m} e^{-n J / k T}}
$$

where $E=H_{K}$. The expectation value in Eq. (3) is average over the lattice and the number of time steps.

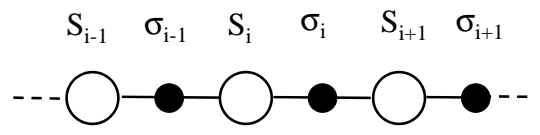

Figure 1: One-dimensional lattice in periodic boundary conditions. Sublattice $A$ and $B$ generate the one-dimensional lattice. Sublattice $A(B)$ is occupied by $S(\sigma)$.

The field cooling $(F C)$ process and the zero-field cooling $(Z F C)$ process for $\mathrm{Tb}$ (III)-Cr (III) are carried out using the cooling and the heating algorithms of CA [36-40]. The cooling and the heating algorithms are divided into two basic parts, the initialization procedure and the taking of measurements. In the initialization procedure, firstly, all the spins in the lattice sites take ferrimagnetic ordered structure $\left(\uparrow(3), \downarrow\left(-\frac{3}{2}\right)\right)$ and the kinetic energy is given to a certain percentage of the lattice via the second variables in the black sites such that the kinetic energy of the site is equal to the change in the Ising spin energy for any spin flip. The values of the kinetic energy per site is set to obtain disordered 
spin configuration for zero field at high temperature. This configuration is run during the 20000 cellular automaton time steps. In the next step, the last configuration in the disordered structure at high temperature was chosen as a starting configuration for the $F C$ and $Z F C$ simulations. Rather than resetting the starting configuration at each energy, it was convenient to use the final configuration at a given energy as the starting point for the next.

\subsection{FC and ZFC Processes}

In the measurement step of the $F C$ algorithm, the last configuration of the initialization procedure in the disordered structure is taken as a starting configuration. The spin system is cooled for a value of non-zero field $\left(h=\frac{H}{J} \neq 0\right)$. During the cooling cycle, a certain amount of energy per site are subtracted from the lattice through the second variable $\left(H_{K}\right)$ after the 2000000 cellular automaton steps. In the zero-field cooling process $(Z F C)$, the initial configuration in the disordered structure is used as a starting configuration for the cooling run at zero-field $(h=0)$. The last configuration at low temperature of the cooling process is taken as a starting configuration for the heating run of the $Z F C$. Then the spin system is heated for a value of non-zero field $\left(h=\frac{H}{J} \neq 0\right)$. During the heating cycle, a certain amount of energy per site is given to the lattice through the second variable $\left(H_{K}\right)$ after the 2000000 cellular automaton steps. These energy amounts are determined considering the $d H_{I}$ values for the possible spin configurations. Thus, the whole energy is used by the spin system. As a result, the spin system does not contain the remnant energy, which affects the temperature measurement.

\section{Results and Discussions}

All simulations were carried out using the cooling and the heating algorithms improved from CCA for the one-dimensional spin 3 - spin $3 / 2$ Ising model. The thermodynamic quantities (the order parameter $(M)$, the susceptibility $(\chi)$, the internal energy $(U)$, and the specific heat $(C / k)$ ) were computed over the lattice and over the number of time steps (2000000) after the discard of the first 100000 time steps during the development of the cellular automaton. Thus, the values of the thermodynamic quantities correspond to the equilibrium average values. The calculations were repeated by field cooling $(F C)$ and the zero-field cooling $(Z F C)$ processes on one-dimensional lattices with the linear dimensions $L=100,500,1000,5000,10000,50000$, and 100000 for periodic boundary conditions.

The thermodynamic quantities are calculated from

$$
\begin{gathered}
M=\frac{1}{N} \sum_{i} S_{i}-\frac{1}{N} \sum_{j} \sigma_{j} \\
U=\frac{1}{H_{0}}\left(\left(\sum_{<i j>} S_{i} \sigma_{j}\right)-\frac{H}{J} \sum_{i}\left(S_{i}+\sigma_{j}\right)\right)
\end{gathered}
$$



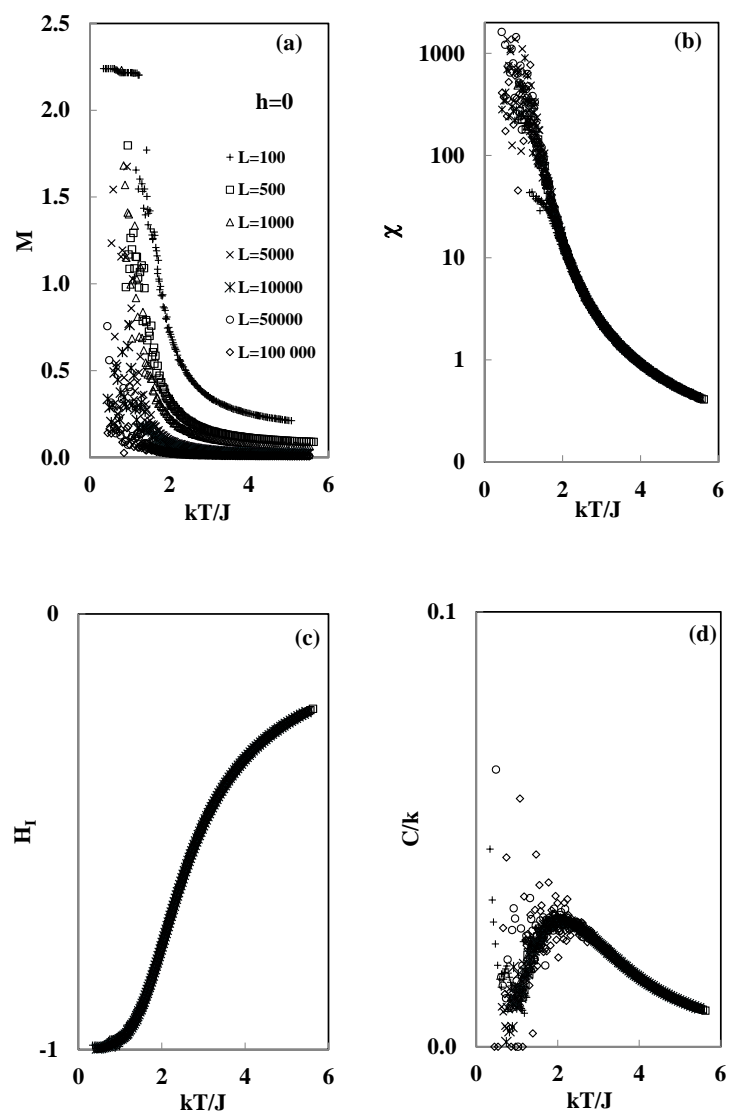

Figure 2: Temperature dependence of (a) Magnetic order parameter $(M)$, (b) Susceptibility $(\chi),(\mathrm{c})$ Internal energy $(U)$, and (d) Specific heat $(C / k)$ at $h=$ $H / J=0$ on $L=100,1000,5000,10000,50000$, and $L=100000$. 


$$
\begin{gathered}
\chi=N \frac{\left\langle M^{2}\right\rangle-\langle M\rangle^{2}}{k T} \\
C / k=N \frac{\left\langle U^{2}\right\rangle-\langle U\rangle^{2}}{(k T)^{2}}
\end{gathered}
$$

where $H_{0}$ is the ground state energy at $k T / J=0$.

\subsection{The behavior of the one-dimensional spin system in the absence of an external magnetic field $\left(h=\frac{H}{J}=0\right)$}

The temperature dependence of the order parameters $(M)$, the susceptibility $(\chi)$, the internal energy $(U)$, and the specific heat $(C / k)$ in the absence of magnetic field $(h=0)$ were illustrated in Fig. 2 for the cooling algorithm. As it is seen in Fig. 2(a), the value of $M$ increases with decreasing temperature $(k T / J \rightarrow 0)$ for each lattice size similar to the one-dimensional Ising model. However, the susceptibility diverges to infinity at absolute zero temperature for all lattice sizes (Fig. 2(b)). At the same time, $U$ does not have an inflection indicating any phase transition and $C / k$ exhibits a broad peak. The critical behavior of $M, \chi, U$, and $C / k$ are compatible with the behavior of the characteristic one-dimensional Ising model, and the isolated one-dimensional Ising chain results which has very weak interchain coupling in 3-d dimensional lattice $[42]$.

The evolution of the magnetization for each lattice was plotted in Fig. 3 as a function of lenght per 200 sites $(L / 200)$ for $k T / J=0.8$ at $h=0$. As it is seen, there are local order regions which are separated by the fluctuations between pozitive and negative magnetization values for $L \geq 10000$ lattices. The spatial fluctuations decrease with decreasing lattice size. This causes an increase in the order parameter at low temperatures for decreasing lattice sizes. Therefore, the one-dimensional Ising model can be modeled in only large lattice size as $L \geq 10000$. In this study, the lattice size was selected as $L=100000$ for the simulation of the one-dimensional Ising model

For $L=100000$, spatial behavior of order parameter are shown in Fig. 4 for $k T / J=0.446,0.808,1.014$, and 1.510 at $h=0$. At $k T / J>0$, the magnetization fluctuates between the local regions for all temperature values. The fluctuations are more often with increasing temperature and so the local order regions disappear as expected for the one-dimensional Ising model.

\subsection{The behavior of the one-dimensional spin system in an external magnetic field $\left(h=\frac{H}{J} \neq 0\right)$}

For $h=0.1$, the evolution of the magnetization was plotted as a function of lenght per 200 sites $(L / 200)$ for different temperatures $(k T / J=0.439,2.264$, 2.499 and 3.513) in Fig. 5. As it is seen, the local order regions do not occur through the lattice for $k T / J \neq 0$. For $k T / J<2.499$, the system shows a 


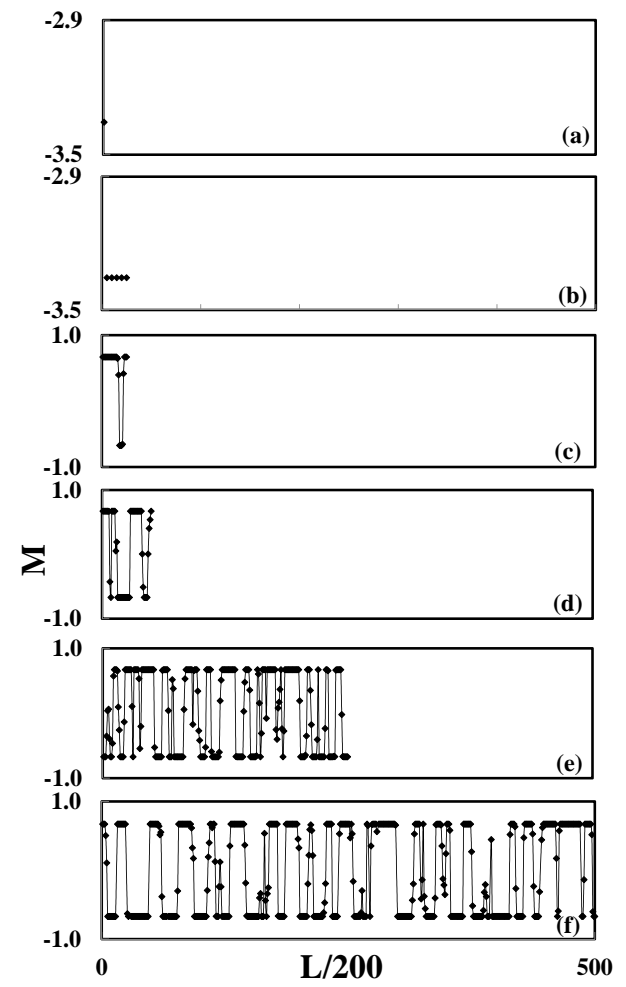

Figure 3: Magnetic order parameter as a function of lenght per 200 sites $(L / 200)$ for $h=\frac{H}{J}=0$ on (a) $L=100$, (b) $L=1000$, (c) $L=5000$, (d) $L=10000$, (e) $L=50000$, and (f) $L=100000$ at $k T / J=0.8$. 


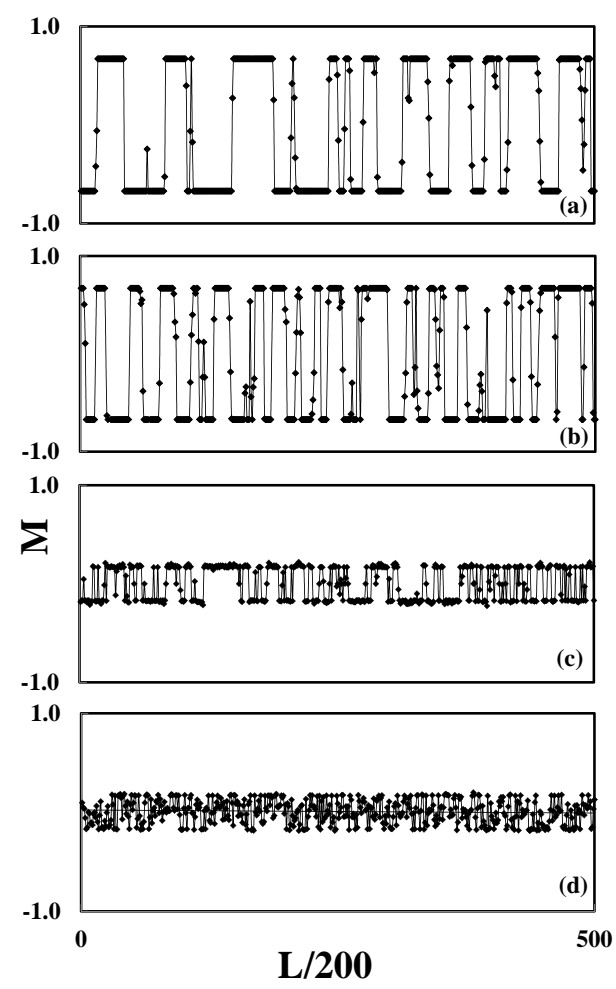

Figure 4: Magnetic order parameter as a function of lenght per 200 sites $(L / 200)$ for $h=\frac{H}{J}=0$ at (a) $k T / J=0.446$, (b) $k T / J=0.808$, (c) $k T / J=1.014$, and (d) $k T / J=1.510$ on $L=100000$. 
long range order $(L R O)$ with the effect of the external magnetic field. On the other hand, the $L R O$ begins to decay at a critical temperature and disappears at high temperatures. This behavior indicates a phase transition depending on the temperature. The temperature dependence of the magnetization was obtained by field cooling $(F C)$ process and zero field cooling $(Z F C)$ process in the interval $0 \leq h \leq 3.4$.

In Fig. 6(a) and 6(b), the temperature variation of $M$ and $\chi$ are illustrated for $Z F C$ and $F C$ processes at $h=0.4$. The values of magnetization for $Z F C$ and $F C$ are different than each other at low temperatures. This difference disappears at high temperatures (Fig. 6(a)). The susceptibilities obtained with $Z F C$ and $F C$ processes have a peak at the same temperature (Fig. 6(b)). The simulation results are in agreement with the experimental cyano-bridged terbium (III)-chromium (III) bimetallic quasi-one dimensional assembly result [4]. In Fig. 6(c), the temperature variations of the magnetizations for $Z F C$ and $F C$ are shown for several magnetic field values. As it seen in the figure, the difference between magnetizations of the $F C$ and the $Z F C$ disappears with increasing $h$ value at low temperatures. Using the $Z F C$ and $F C$ magnetization values, the hysteresis curves were obtained for $L=100000$. In Fig. 7 (a) and 7 (b), hysteresis curves are illustrated for $k T / J=1.5$ and 2 temperature values. As it is seen from hysteresis curves, the spin system has remnant magnetization when the magnetic field drops to zero. The remnant magnetization values are estimated from the hysteresis curves for several temperatures and shown in Fig. 7(c). The temperature variation of remnant magnetization is similar to spontaneous magnetization of a magnetic system above one dimension.

\section{Conclusion}

The mixed spin 3- spin $3 / 2$ Ising model with antiferromagnetic nearest-neighbor interaction is simulated on one-dimensional lattices with linear dimension $L=$ 100, 500, 1000, 5000, 10000, 50000, and 100000 using Cellular Automaton cooling and heating algorithms improved from Creutz Cellular Automaton (CCA). The values of order parameter $(M)$, susceptibility $(\chi)$, Internal energy $(U)$ and specific heat $\left(C / k_{B}\right)$ are calculated using cooling and heating algorithms $\left(h=\frac{H}{J}=0\right)$. The mixed spin system shows the one-dimensional Ising chain behavior for $h=0$ [42]. At the same time, the system exhibits a long-range order $(L R O)$ at low temperatures on the one-dimensional lattice in the presence of the external magnetic field $\left(h=\frac{H}{J}=0.1\right)$. The hysteresis curves are obtained from zero field cooling $(Z F C)$ and field cooling $(F C)$ magnetization values in the presence of the external magnetic field $(h \neq 0)$. It is seen that the mixed spin 3- spin 3/2 Ising model has remarkable remnant magnetization as presented in the experimental study [4]. The results of the one-dimensional Ising model with antiferromagnetic nearest-neighbor interaction are similar with cyano-bridged $\mathrm{Tb}$ (III) - Cr (III) bimetallic quasi-one dimensional assembly $\left(\left[\mathrm{Tb}\left(\mathrm{H}_{2} \mathrm{O}\right)_{2}(\mathrm{DMF})_{4}\left\{\mathrm{Cr}(\mathrm{CN})_{6}\right\}\right] \cdot \mathrm{H}_{2} \mathrm{O}(\mathrm{DMF}=\right.$ dimethylformamide)) results [4]. As a result, the magnetic behavior of the cyano-bridged 


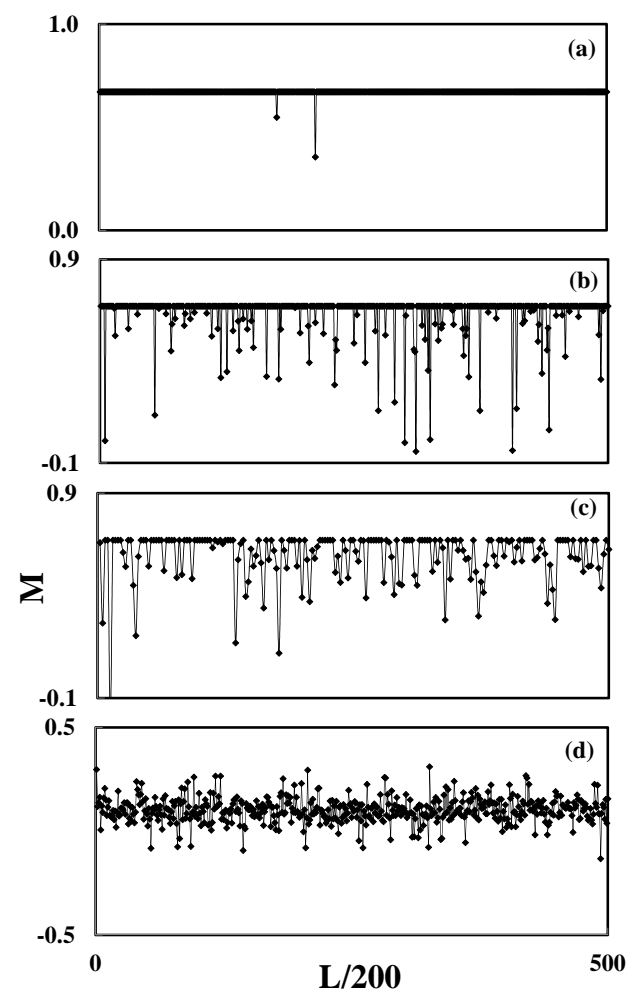

Figure 5: Magnetic order parameter as a function of lenght per 200 sites $(L / 200)$ for $h=\frac{H}{J}=0.1$ at (a) $k T / J=0.439$, (b) $k T / J=2.264$, (c) $k T / J=2.499$ and (d) $k T / J=3.513$ on $L=100000$. 

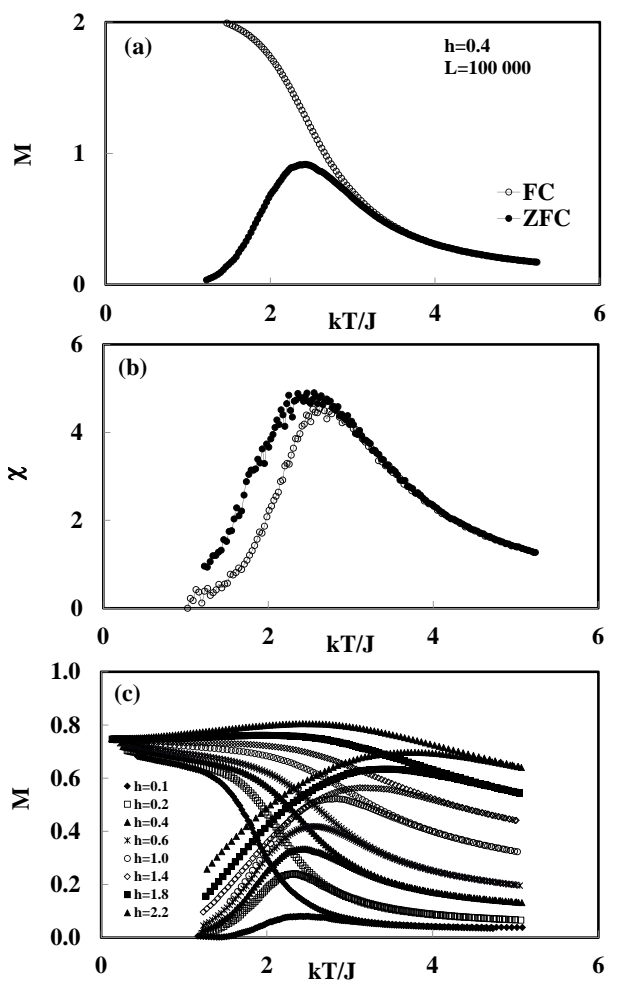

Figure 6: The temperature dependences of (a) the magnetization $(M)$, and (b) the susceptibility $(\chi)$ for $h=\frac{H}{J}=0.4$. (c) The temperature dependences of the magnetization $(M)$ in the interval $0 \leq h \leq 3.4$. The thermodynamic quantities are obtained by $F C$ process and $Z F C$ process on $L=100000$. 

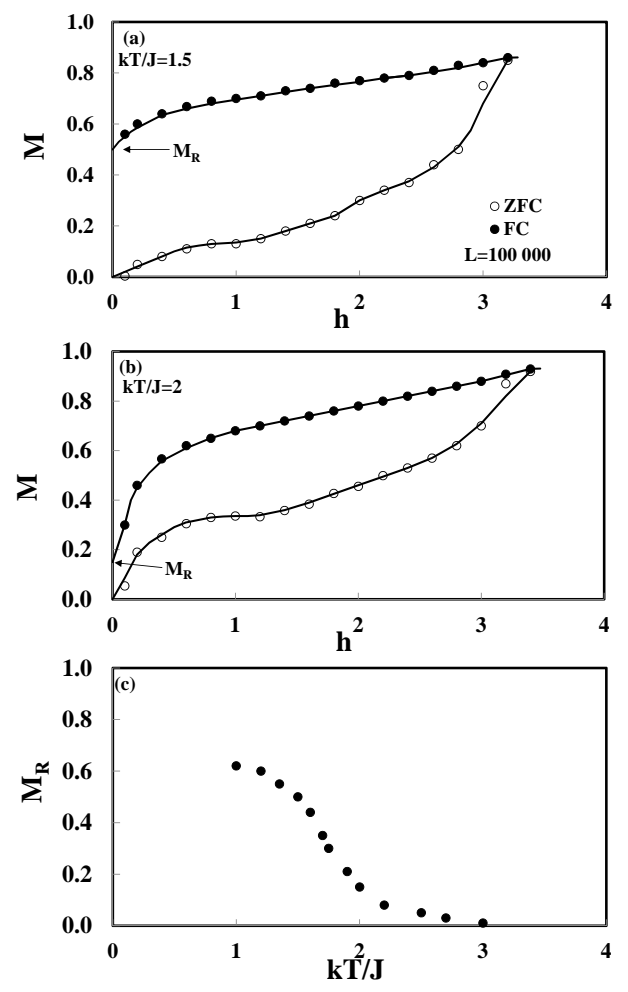

Figure 7: The hysteresis curves obtained using the zero field cooling $(Z F C)$ and the field cooling $(F C)$ magnetization measurements on $L=100000$ at (a) $k T / J=1.5$, (b) $k T / J=2.0$, and (c) The temperature dependences of remnant magnetization $\left(M_{R}\right)$. 
Tb (III) - Cr (III) bimetallic assembly can be determined by intrachain nearestneighbor interactions in the absence of interchain interaction on one-dimension. Thus, the cyano-bridged Tb (III) - Cr (III) bimetallic assembly may be considered as one dimensional instead of quasi one dimensional spin system. Our calculations show that the high temperature phase transition on a one-dimensional mixed spin 3- spin 3/2 system, similar to the cyano-bridged Tb (III) - Cr (III) bimetallic assembly, is caused by the ferrimagnetic nature of the spin system.

\section{References}

[1] R.J. Baxter, Exactly solved models in statistical mechanics (Academic press inc., San Diego, 1989).

[2] K.S. Asha, K.M. Ranjith, A. Yogi, R. Nath, S. Mandal, Dalton Trans. 44, 19812 (2015).

[3] J. M. Law, H.-J. Koo, M.-H. Whangbo, E. Brücher, V. Pomjakushin, and R. K. Kremer, Phys. Rev. B 89, 014423 (2014).

[4] Y. Guo, G.-F. Xu, C. Wang, T.-T. Cao, J. Tang, Z.-Q. Liu, Y. Ma, S.-P. Yan, P. Cheng, D.-Z. Liao, Dalton Trans. 41, 1624 (2011).

[5] X. Zhang, G. Zhang, T. Jia, Y. Guo, Z. Zeng, H.Q. Lin, Phys. Lett. A 375, 2456 (2011).

[6] S. Shiraki, J of Vacuum Soc. of Japan 52, 595 (2009).

[7] M. Estrader, J. Ribas, V. Tangoulis, X. Solans, M.Font-Bardia, M. Maestro, C. Diaz, Inorg. Chem. 45, 8239 (2006).

[8] A. Figuerola, C. Diaz, M.S.El Fallah, J. Ribas, M. Maestro, J. Mahia, Chem. Commun. 13, 1204 (2001).

[9] F. Hulliguer, M. Landolt, H. Vetsch, J. Solid State Chem. 18, 283 (1976).F. Hulliger, M. Landolt, H. Vetsch

[10] Y.-Z. Zhang, G.-P. Duan, O. Sato, S. Gao, J. Mater. Chem. 16, 2625 (2006).

[11] H. Zhou, A.-H. Yuan, S.-Y. Qian, Y. Song, G.-W. Diao, Inorg. Chem. 49, 5971 (2010).

[12] T. Hozumi, S.-̇̇. Ohkoshi, Y. Arimoto, H. Seino, Y. Mizobe, K. Hashimoto, J. Phys. Chem. B 107, 11571 (2003).

[13] H. Svendsen, J. Overgaard, M. Chevallier, E. Collet, Y.-S. Chen, F. Jensen, B.B. Iversen, Chem. Eur. J. 16, 7215 (2010).

[14] S.-̇̇. Ohkoshi, K.-İ. Arai, Y. Sato, K. Hashimoto, Nat. Mater. 3, 857 (2004).

[15] A. Dakhama and N. Benayad, J.of Magn. and Magn.Mater. 213, 117 (2000).

[16] M. Azhari, N. Benayad, M. Mouhib, Superlattices and Microstructures 79, 96 (2015).

[17] G.M. Buendia and M.A. Novotny, J. Phys. Condens. Matter. 9, 5951 (1997)

[18] E. Aydiner, Y. Yuksel, E. Kis-Cam, H. Polat, J.of Magn. and Magn.Mater. 321, 3193 (2009). 
[19] W. Selke, J. Oitmaa, J.Phys: Condens. Matter. 22, 076004 (2010).

[20] F.W.S. Lima, M.A. Sumour, Physica A 391, 948 (2012).

[21] A. Zaim and M. Kerouad, Physica A 389, 3435 (2010).

[22] E. Albayrak, Solid State Communications 159, 76 (2013). (2002).

[23] Y. Nakamura, J.W. Tucker, IEEE Transactions on magnetics 38, 2406

[24] A. Bobák, O.F. Abubrig and D. Horváth, J.of Magn. and Magn. Mater. 246, 177 (2002).

[25] M. Ertaş, M. Keskin, Physics Letters A 379, 1576 (2015).

[26] B. Deviren, M. Batı and M. Keskin, Phys. Scr. 79, 065006 (2009).

[27] W.Guo-Zhu, M.Hai-Ling, Commun.Theor. Phys. 51, 756 (2009).

[28] B. Deviren, Y. Polat, M. Keskin, Chin. Phys. B 20, 060507 (2011).

[29] W. Jiang, W. Wang, F. Zhang and W.J. Ren, J.of Appl. Phys. 105 , 07E321 (2009).

[30] Y.Kai-Lun, L.Jian-Wen, L.Zu-Li, F.Hua-Hua and Z. Lin, Theor. Phys. 47, 741 (2007).

[31] M. Ertaş, M. Keskin, B. Deviren, Physica A 391, 1038 (2012).

[32] S.G. Carling, P. Day, Polyhedron 20, 1525 (2001).

[33] Y. Nakamura, J.Phys. Condens. Matter. 12, 4067 (2000).

[34] M. Charilaou, K.K. Sahu, A.U. Gehring and J.F. Löffler, Phys. Rev. B 84, 224434 (2011).

[35] B. Deviren, M. Keskin, O. Canko, J.of Magn. and Magn.Mater. 321, 458 (2009).

[36] A. Özkan, Phase transitions 89, 94 (2016).

[37] A. Özkan, B. Kutlu, Cent. Europ. J. of Phys. 9, 884 (2011).

[38] A. Özkan, B. Kutlu, Int. J. of Mod. Phys. C 20, 1617 (2009).

[39] B. Kutlu, A. Özkan, N. Seferoğlu, A. Solak and B. Binal, Int. J. Mod. Phys.C 16, 933 (2005).

[40] N. Seferoğlu, B. Kutlu, Physica A 374, 165 (2007).

[41] M. Creutz, Ann. Phys. 167, 62 (1986).

[42] T. Graim, D.P. Landau, Phys. Rev. B 24, 5156 (1981). 